\title{
Changes in assumption of care and primary drug use in substance use in pregnancy service
}

\author{
Duong $C^{1 *}$, Drury $A^{1}$, Perry $N^{1,2}$, Brown $A^{1,2}$, Ling $S^{1,2}$, Lawson $D^{1}$, Byrne $J^{1}$, Keats $J^{1}$,Nean $M^{1}$ and Dunlop $A J^{1,2,3}$ \\ ${ }^{1}$ Hunter New England Local Health District, NSW, Australia \\ ${ }^{2}$ University of Newcastle, NSW, Australia \\ ${ }^{3}$ Hunter Medical Research Institute, NSW, Australia
}

\begin{abstract}
Introduction/Issues: Substance Use in Pregnancy services exists in most Health Districts in NSW to improve antenatal care and postnatal referrals for pregnant women who use drugs. The John Hunter Hospital is a level 6 tertiary referral hospital.

Method/Approach: A seven year (2010-2016) retrospective medical record audit of the John Hunter Hospital Substance Use in Pregnancy Service was performed. Number of referrals, assumption of care, primary drug of concern, patients on opioid pharmacotherapy, fetal death and miscarriages were recorded.

Key findings: Over the seven year period a total of 539 referrals were received for pregnant women, mean age 28.6 years (range 15-43). Referrals increased during the period (48 in 2010, 88 in 2016). Analysis of assumption of care at birth revealed a total of 134 removals (24.9\%). Assumptions of care during the 2010-2014 period remained consistently (26-29.1\%), but reduced from $27.4 \%$ in 2014 (23/84) to $17.4 \%$ in 2015 (16/92, Fisher's exact 0.54 , p<0.01). Changes in primary drug occurred over time, prescribed opioid agonist treatment decreased (66.7\% in 2010, 21.6\% in 2016), cannabis (10.4\% in 2010, $45.5 \%$ in 2016$)$ and amphetamines (10.4\% in 2010, 34.1\% in 2016) increased. Women presenting with alcohol use was less common (7.4\% of all referrals). Tobacco use was almost universal but cessation infrequent. Fetal death and miscarriage/termination was uncommon $(1.5 \%, 4.5 \%)$.
\end{abstract}

Discussions and conclusions: An increase number of referralsto the drugs in pregnancy service has occurred alongside a change in the profile of primary drug use, with an overall increase in referrals of women who use cannabis and amphetamines. The reduction in assumptions of care at birth may relate to policy changes by Family and Community Services in 2015 including the use of Parental Responsibility Contracts.

\section{Introduction}

Use of psychoactive substances in pregnancy can have a detrimental effect on maternal and fetal morbidity and mortality[1].Fetal adverse outcomes include "premature birth, low birthweight, and fetal death in utero and require admission to neonatal intensive or special care nurseries" [2]prenatally. Outcomes may be complicated by multiple concurrent drug use problems (including tobacco dependence, alcohol, opioid, stimulant or other drug use problems), co-occurring maternal medical and mental health issues, poor self-experience of parenting and a range of psychosocial factors including intimate partner violence, an unstable social environment (including unstable housing) and socioeconomic factors (poverty, legal problems, lack of support).

The role of a Substance Use in Pregnancy and Parenting Service (SUPPS) is to provide comprehensive care as part of an antenatal team to actively engage pregnant women in antenatal care and substance use treatment. The John Hunter Hospital (JHH) is a level 6 tertiary hospital in regional NSW Australia and has a SUPPS which was first established in 1983 at the Calvary Mater Hospital before relocating to the JHH in 1991. The service is staffed by midwives, obstetricians, social workers, child protection workers, drug and alcohol (D\&A) hospital consultation liaison and community based clinicians including nursing and addiction medicine specialists.

The service is integrated into routine antenatal care and available to all pregnant women who disclose or have a significant risk of current or recent substance use (other than tobacco alone). There are multiple points of referral to the specialised clinic with each patient receiving a comprehensive multidisciplinary assessment and collaborative treatment plan which includes substance use treatment (i.e., counselling, withdrawal, pharmacological treatment), case management (i.e., women's health, mental health, psychosocial, child safety, parenting, accommodation) and infant health needs (i.e.,fetal monitoring, screening and treatment for neonatal abstinence syndrome and/or fetal alcohol spectrum disorder).

Where there is on-going or substantial evidence that the newborn is at risk of serious harm, involvement by child protective services occurs. We conducted a chart audit to describe current patterns of drug use, care provided and document assumptions of care for women attending this service from 2010 to 2016 .

\section{Methods and materials}

\section{Design and participants}

The current study is a retrospective clinical audit of electronic medical records relating to participants who were seen in the SUPPS

Correspondence to: Chi Duong, Hunter New England Local Health District, NSW, Australia, Tel: 024016 4514; E-mail: Chi.Duong@hnehealth.nsw.gov.au

Key words: child protection, substance related disorders, pregnancy

Received: June 12, 2017; Accepted: June 26, 2017; Published: June 30, 2017 
at John Hunter Hospital between $1^{\text {st }}$ January 2010 to $31^{\text {st }}$ December 2016. Ethics approval for the study was obtained from the Hunter New England Local Health District Human Research Ethics Committee (AU201605-01).

The data set comprised information on 539 women who had reported use of psychoactive substance(s) (other than tobacco alone) to midwives, obstetricians, social workers and/or allied health staff within the antenatal clinics and who had subsequently attended SUPPS at $\mathrm{JHH}$ as well as information of their newborns up until point of birth.

Inclusion criteria included pregnant women who had been referred and had attended the SUPPS between the above dates. Exclusion criteria included those who had given birth either prior to or after the specified timeframe, women who had been referred to SUPPS but had not ever attended an antenatal appointment and women who were referred but had treatment elsewhere (i.e., GP prescriber or another specialised clinic).

\section{Maternal data}

The data collected on each woman was their age at presentation, primary substance used, current opioid agonist treatment (i.e., methadone or buprenorphine) and overall number of referrals.

For women who reported multiple substances used, the treating SUPPS clinician coded primary substance use based upon referral request and principal substance use problem.

\section{Fetal data}

Perinatal data collected on each neonate was the number of fetal deaths, termination of pregnancies, miscarriages and presence of neonatal abstinence syndrome and diagnosis of fetal alcohol spectrum disorder.

\section{Statistical analysis}

All data collected was securely stored on secure computers within the Drug and Alcohol Service. This information was then de-identified and inputted into a MS Excel spreadsheet.

Statistical analysis was conducted. Means and proportions were calculated. Fischer's exact test was performed to compare prevalence of assumptions of care using SPSS software.

\section{Results}

\section{General demographics}

The mean age of women presenting across the 7 years was 28.6 years. The median age was 28 with a mode of 27 . The range was between 15 years of age and 43 years of age. The overall number of referrals to SUPPS was 539. This represents a 1.8 fold increase from 48 in 2010 to 88 in 2016.

\section{Assumptions of care}

There were a total of 134 infants removed from parental care at birth, representing $24.9 \%$ (134/539). The rate of assumptions of care remained consistent for the period 2010 to 2014 (26.0\%-29.1\%). However, it was noted that there was a decrease to $17.4 \%$ in 2015 (Fisher's exact score $0.54, \mathrm{p}<0.01$ ). This has subsequently increased to $21.6 \%(19 / 88)$ in 2016. Table 1 indicates the numbers of assumptions of care (blue) in direct comparison with the total number of referrals per year. Despite the number of referrals continuing to increase, the numbers of assumptions has remained relatively consistent.

\section{Primary substance}

Patterns of primary substance changed over the period 2010 to 2016. Table 2 shows that opioid as primary substance has decreased from $67 \%$ in 2010 to $22 \%$ in 2016 . Conversely, cannabis use has significantly increased from $10 \%$ in 2010 to $46 \%$ in 2016 . There has also been a moderate increase in reported amphetamine use from $10 \%$ in 2010 to $34 \%$ in 2016. Alcohol use remained low at $13 \%$ in 2010 and $10 \%$ in 2016.

Table 3 clearly shows a decline in the proportion of pregnant women referred for opioids as their primary substance and a significant increase in both cannabis and amphetamines with alcohol use remaining below $15 \%$ annually.

\section{Discussion}

\section{Assumption of care}

Disclosures to child protection services by health professionals of pertinent concerns regarding the welfare of a neonate are often a contentious and sensitive issue which may jeopardise the therapeutic relationship between the patients attending the clinic and SUPPS clinicians. In 2015, the Child Protection Legislation Amendment Act 2014 was introduced which sought to expand Parental Responsibility Contracts to include expectant women, court issued Parental Capacity Orders and streamlining of open adoptions in the context of NSW having the highest rate of child protection notifications (61\%) and the highest level of child protection activity[3].The implementation of this amendment Act (enacted in March 2015) appears to coincide temporarily with a decrease in the numbers of assumption of care decrease noted in $2015(17.4 \%, 16 / 92)$ at JHH which may be attributed to the working relationship that exists between child protection services (such as FAC and Mayfield High Risk infant team) and community outpatient teams. In addition, there is continued support for new mothers from midwives and social workers for a period of 4-8 weeks postpartum to provide monitoring for the babies' general growth

Table 1. No. of referrals and assumption of care at birth.

\begin{tabular}{|l|l|l|l|l|l|l|l|}
\hline $\begin{array}{l}\text { Number of referrals, } \\
\text { total yearly births and } \\
\text { Assumptions of care at } \\
\text { birth (AoC) }\end{array}$ & $\mathbf{2 0 1 0}$ & $\mathbf{2 0 1 1}$ & $\mathbf{2 0 1 2}$ & $\mathbf{2 0 1 3}$ & $\mathbf{2 0 1 4}$ & $\mathbf{2 0 1 5}$ & $\mathbf{2 0 1 6}$ \\
\hline Total births at JHH & 3826 & 4113 & 4132 & 4112 & 4020 & 3973 & 4054 \\
\hline Referrals & 48 & 68 & 73 & 86 & 84 & 92 & 88 \\
\hline Assumptions of care at birth & 13 & 19 & 19 & 25 & 23 & 16 & 19 \\
\hline
\end{tabular}

Table 2. Number of referrals (\% of all referrals).

\begin{tabular}{|c|c|c|}
\hline & $\mathbf{2 0 1 0}$ & $\mathbf{2 0 1 6}$ \\
\hline $\begin{array}{c}\text { Prescribed Opioid Agonist } \\
\text { Treatment }\end{array}$ & $66.7 \%(32 / 48)$ & $21.6 \%(19 / 88)$ \\
\hline Cannabis & $10.4 \%(5 / 48)$ & $45.5 \%(40 / 88)$ \\
\hline Amphetamine & $10.4 \%(5 / 48)$ & $34.1 \%(30 / 88)$ \\
\hline Alcohol & $12.5 \%(6 / 48)$ & $10.2 \%(9 / 88)$ \\
\hline
\end{tabular}

Table 3. Primary substance by year.

\begin{tabular}{|l|l|l|l|l|l|l|l|}
\hline $\begin{array}{l}\text { Primary Substance by } \\
\text { Year }\end{array}$ & $\mathbf{2 0 1 0}$ & $\mathbf{2 0 1 1}$ & $\mathbf{2 0 1 2}$ & $\mathbf{2 0 1 3}$ & $\mathbf{2 0 1 4}$ & $\mathbf{2 0 1 5}$ & $\mathbf{2 0 1 6}$ \\
\hline Cannabis & $5 / 48$ & $12 / 68$ & $19 / 73$ & $24 / 86$ & $28 / 84$ & $44 / 92$ & $40 / 88$ \\
\hline $\begin{array}{l}\text { Prescribed Opioid } \\
\text { Agonist Treatment }\end{array}$ & $32 / 48$ & $39 / 68$ & $28 / 73$ & $39 / 86$ & $32 / 84$ & $26 / 92$ & $19 / 88$ \\
\hline Amphetamines & $5 / 48$ & $12 / 68$ & $14 / 73$ & $18 / 86$ & $22 / 84$ & $17 / 92$ & $30 / 88$ \\
\hline Alcohol & $6 / 48$ & $5 / 68$ & $10 / 73$ & $5 / 86$ & $2 / 84$ & $3 / 92$ & $9 / 88$ \\
\hline $\begin{array}{l}\text { Other [dexamphetamine } \\
\text { (3), cocaine (1)] }\end{array}$ & $0 / 48$ & $\begin{array}{l}1 / 68 \\
\text { (cocaine) }\end{array}$ & $0 / 73$ & $\begin{array}{l}2 / 86 \\
\text { (dex) }\end{array}$ & $\begin{array}{l}1 / 84 \\
\text { (dex) }\end{array}$ & $0 / 92$ & $0 / 88$ \\
\hline
\end{tabular}


and development as well as assessment of NAS (neonatal abstinence syndrome). However, this rate of assumptions of care has increased to $21.6 \%(19 / 88)$ in 2016 . This may be a reflection of an increasing number of vulnerable women in this population group being seen at SUPPS with 2015 being an anomaly rather than a true representation of the effects of policy changes.

Taplin found a strong correlation between the number of assumptions of care at birth and substance use in pregnancy[4]as well as involvement with child protection services[5]. Clinically, pregnant women who were previously reviewed in SUPPS and had their children assumed at birth were flagged for re-referral to SUPPS once they fall pregnant again. Underpinning this is the complex interplay between psychosocial factors, medical and mental health comorbidities and substance use that coexists in pregnant women.

\section{Substance use}

There is no state or national routine dataset available to compare referrals and patterns of substance use by pregnant women. The National Drug Household Survey does collect data on self-reported drug use during pregnancy [1].The 2013 survey recorded self-reported use (i.e., any recent substance use), after becoming aware of the pregnancy, of tobacco (10\%), alcohol (26\%), prescription analgesics $(0.9 \%)$ and all illicit drugs (1.6\%). This survey may under-estimate drug use during pregnancy, and not all use results in problematic use requiring treatment, however, it is worth noting the trend in presentations and substances used in our audit is vastly different from these data.

There appears to be a significant reduction in opioid related presentations which may reflect changes in patterns of substance use in this particular cohort and/or a reflection of an ageing cohort of patients who are currently on opiate agonist treatment. This is in agreement with the 2013 National Drug Strategy Household Survey which found "illicit drug use remained stable ... [but] ... there was a significant change for a few specific drugs" including recent use of heroin which fell from $0.2 \%$ to $0.1 \%[6]$.

In direct contrast, there appears to be a significant increase in both cannabis and amphetamine use. Increased cannabis may relate to better identification of cannabis associated harm and/or a reflection of its increased popularity of 'medical' cannabis use in this population[7]. Increased amphetamine use may be related to increased availability and lower cost[8].The upward trends for amphetamine and cannabis use suggest the need for increased therapeutic support and resources to accommodate increasing demand for counselling services.

Disclosure of alcohol use remains low potentially due to the stigma which is associated with fetal alcohol syndrome disorder.

\section{Summary and conclusion}

At present, SUPPS involvement with pregnant women at John Hunter Hospital ceases at the time of discharge following the birth of the neonate. In many instances, re-engagement occurs only if these patients become pregnant again. In many instances what may be seen clinically is a loss of motivation and return to their "normal" pattern of substance use at the time of re-engagement in SUPPS. Often a sense of resignation or resistance is met by SUPPS clinicians who attempt to establish rapport with these patients in order to ascertain a baseline indication of substance use.

Postnatal follow up services would significantly help to support, review, and treat these patients, their partners and their newborn with a view to implement longer term follow-up to address psychosocial risks perhaps as a link up community specialised clinic to facilitate easier access.

Currently, data collection is completed independently by antenatal clinics with no consistent data collection method implemented. This results in duplication and of data collection bias and errors. The introduction of shared information across all antenatal services would aid in the conducting of audits which can be utilised to better improve clinical outcomes for patients.

Multiple concurrent drug use is considered to be the norm[9]in this cohort with many patients commonly reporting tobacco consumption as a secondary substance of use. In this study $65.9 \%(58 / 88)$ of the patients in 2016 reported tobacco consumption with infrequent cessation. A clinical trial of Counselling, Nicotine Replacement Therapy and contingency management trial funded by NSW Health aims to examine a strategy to address this concern[10].

\section{References}

1. Finnegan LP, Kandall SR (2005) Maternal and neonatal effects of alcohol and drugs substance abuse: a comprehensive textbook. (4th edn). PA: Lippincott, Williams \& Wilkins, Philadelphia, USA pp: 805-839.

2. Callaghan T, Crimmins J, Schweitzer D (2011) Children of substance-using mothers child health engagement. J Paediatr Child Health 47: 223-227. [Crossref]

3. Olsen A (2015) Punishing parents: Child removal in the context of drug use. Drug Alcohol Rev 34: 27-30. [Crossref]

4. Taplin S, Mattick RP (2015) The nature and extent of child protection involvement among heroin-using mothers in treatment: High rates of reports, removals at birth and children in care. Drug Alcohol Rev 34: 31-37.[Crossref]

5. McGlade A, Ware R, Crawford M (2009) Child Protection Outcomes for Infants of Substance-Using Mothers: A Matched-Cohort Study. Paediatrics 124: 285-292. [Crossref]

6. AIHW (2014) National Drug Strategy Household Survey detailed report: 2013. Drug statistics series no. 28 Cat. No. PHE 183. Canberra: AIHW.

7. Mather LE, RauwendaalER, Moxham-Hall VL, Wodak AD (2013) (Re)introducing medicinal cannabis. Med J Aust 199: 759-761.[Crossref]

8. http://www.aic.gov.au/publications/current series/rip/21-40/rip36.html

9. KennareR, Heard A, Chan A (2005) Substance use during pregnancy: risk factors and obstetric and perinatal outcomes in South Australia. Aust N Z J Obstet Gynaecol 45: 220-225.[Crossref]

10. http://www.hnehealth.nsw.gov.au/working-together/Pages/Round 1 2016-2017 HNE Health EOI Outcomes.aspx

Copyright: (C2017 Duong C. This is an open-access article distributed under the terms of the Creative Commons Attribution License, which permits unrestricted use, distribution, and reproduction in any medium, provided the original author and source are credited. 